CORRECTION

Check for updates

\title{
Correction: Autophagy receptor CCDC50 modulates STING- mediated interferon response in viral infection and autoimmune disease
}

Panpan Hou, Yuxin Lin, Zibo Li, Ruiqing Lu, Yicheng Wang, Tian Tian, Penghui Jia, Xi Zhang, Liu Cao, Zhongwei Zhou, Chunmei Li, Jieruo Gu and Deyin Guo iD

(c) The Author(s), under exclusive licence to CSI and USTC 2021

Cellular \& Molecular Immunology (2021) 18:2682; https://doi.org/10.1038/s41423-021-00777-7

Correction to: Cellular \& Molecular Immunology https://doi.org/ 10.1038/s41423-021-00758-w

In the version of this article initially published, one unintended error was made during manuscript editing. The title of the article was not in accordance with that of the original manuscript, and the correct statement is "Autophagy receptor CCDC50 modulates STING-mediated interferon response in viral infection and autoimmune disease". The results and conclusions are not affected. 